\title{
Building Meaning in Schizophrenia
}

\author{
Gina R. Kuperberg \\ Department of Psychology, Tufts University, Medford, MA 02155. Department of Psychiatry and \\ the Athinoula A. Martinos Center for Biomedical Imaging, Massachusetts General Hospital, BIdg \\ 149, 13th Street, Charlestown, MA 02129, USA
}

\begin{abstract}
The schizophrenia syndrome is clinically characterized by abnormal constructions of meaning during comprehension (delusions), perception (hallucinations), action (disorganized and non-goaldirected behavior) and language production (thought disorder). This article provides an overview of recent studies from our laboratory that have used event-related potentials and functional magnetic resonance imaging to elucidate abnormalities in temporal and spatial patterns of neural activity as meaning is built from language and real-world visual events in schizophrenia. Our findings support the hypothesis that automatic activity across semantic memory spreads further within a shorter period of time in thought-disordered patients, relative to non-thought-disordered patients and healthy controls. Neuroanatomically, increased activity to semantic associates is reflected by inappropriate recruitment of temporal cortices. In building meaning within sentences, the fine balance between early semantic memory-based and later syntactically-driven processing (dictating 'who does what to whom') is disrupted, such that comprehension is driven primarily by semantic memory-based processes. This imbalance is neuroanatomically reflected by preserved activity within temporal and inferior prefrontal cortices, but abnormal modulation of dorsolateral prefrontal and parietal cortices. In building meaning between sentences (discourse), patients fail to immediately construct coherence links but, perhaps at a later stage of processing, show an inappropriate recruitment of temporal and inferior prefrontal cortices to incoherent discourse, again reflecting inappropriate semantic memory processing (inappropriate inferencing). Finally, these principles may generalize to understanding abnormalities in real-world visual event comprehension in schizophrenia, where patients again show reduced neural activity in determining relationships around goal-directed actions, and comprehension is again dominated by semantic memory-based processes.
\end{abstract}

\section{Keywords}

language; semantic; schizophrenia; comprehension; real-world events; fMRI; ERP; N400; temporal cortex; prefrontal cortex

\section{Introduction}

Clinically, schizophrenia manifests at the highest levels of human function. Yet, over the last two decades, most cognitive paradigms used to study schizophrenia have taken a relative reductionistic approach using stimuli presented out of their every-day contexts (e.g. symbols, numbers, words and faces). A complementary approach - and the one taken by our own laboratory - is to study how imbalances in the operation of multiple interacting systems can lead to abnormalities in the build-up of higher-order meaning in schizophrenia. One way

Correspondence to: Gina R Kuperberg MD PhD, Department of Psychology, Tufts University, or Dept. of Psychiatry, Mass. General Hospital (East), Tel: 617726 3432, Fax: 617812 4799, kuperber@nmr.mgh.harvard.edu. 
in which this can be done is through the study of semantics - how meaning is constructed for comprehension, communication and action in the real world. The important caveat is that this approach can only be instantiated within a theoretical framework of how meaning is normally built up. The aim of this article is to provide an overview of some recent studies from our own lab that have used event-related potentials (ERPs) and functional magnetic resonance imaging (fMRI) to study the cognitive neuroscience of language and semantic processing in healthy individuals and in patients with schizophrenia. For more comprehensive reviews of semantic and language function in schizophrenia, please see references [1] and [2].

\section{The structure and function of semantic memory}

One of the most intuitively compelling theories of the 'loosening of associations' sometimes seen in schizophrenia patients and first described by Bleuler as being fundamental to the schizophrenia syndrome [3], is that it arises from an increased automatic spread of activation ('hyperactivity') across the semantic network $[4,5]$. Testing this hypothesis, however, presents many challenges. This is because schizophrenia patients are impaired in their ability to mobilize and use strategies to perform almost any task, and such impairments can obscure the behavioral effects of any automatic semantic hyperactivity. For example, in a traditional behavioral semantic priming paradigm, healthy individuals show shorter reaction times to recognize target words that are preceded by semantically related words ("tiger"- "stripes") relative to unrelated words ("truck"- "stripes") (reviewed in [6]). This so-called "semantic priming effect' arises, in part, from an automatic spread of activation from the internal representation of the prime to the target, thus facilitating target recognition. However, it also arises because participants use semantic strategies to facilitate target recognition, such as attempting to predict the target or to explicitly match prime and target [6]. Given patients' known deficits in strategic processing, it is therefore not surprising that their behavioral semantic priming effect is often smaller than that of matched controls (reviewed in [1]), even though this observation is inconsistent with the semantic hyperactivity hypothesis of schizophrenia.

To derive a more accurate measure of automatic activity within the semantic network in schizophrenia, one must therefore tease out any strategic influences. We have done this in two ways. The first, building upon previous behavioral [7] and ERP [8] work, aimed to circumvent such strategies altogether $[9,10]$. We separated the prime and target by a short interval that did not allow time for strategic processing; we introduced targets that were not only directly related but also indirectly linked to their primes through an unseen mediator, e.g. "lion" (tiger) "stripes" (predictive and matching strategies are ineffective in priming such indirectly-related targets); finally, rather than requiring participants to make behavioral decisions on each trial, we measured the modulation of the N400 event-related potential (ERP) - a brain potential that indexes the earliest stage of integrating meaning in the brain (between 300-500msec post target onset) [11] - as an index of semantic priming; (to ensure deep semantic processing, participants monitored for a semantic category in probe filler items). We demonstrated that patients with thought disorder exhibited a larger early indirect N400 priming effect (between 300-400msec) than patients without thought disorder or healthy controls [10]. This finding supports the hypothesis that activation spreads further within a shorter period of time in specific association with positive thought disorder in schizophrenia.

The second way in which we have partially teased apart controlled strategic and automatic semantic processes in schizophrenia is to use fMRI which, unlike ERPs, has the spatial resolution to reveal some neuroanatomic segregation between these different types of semantic processes. Controlled semantic processing is thought to be mediated by inferior 
prefrontal cortices, while more automatic or implicit semantic associative processing is thought to take place primarily within temporal-occipital cortices; moreover, activity within prefrontal and temporal regions can be, at least partially, experimentally dissociated during semantic priming in healthy individuals [12]. fMRI measures may also be more sensitive than ERPs to the underlying mechanisms of priming, rather than only its effects. In schizophrenia patients, we demonstrated abnormal increases in hemodynamic activity within temporal cortices to indirectly related (versus unrelated) word-pairs in schizophrenia [13]. These abnormal increases correlated with severity of positive thought disorder. We interpreted such increases as reflecting associative hyperactivity in schizophrenia [13]. Future studies will determine whether such hyperactivity is purely automatic in nature.

\section{Building up meaning within sentences}

Encountering words within sentences leads to activation not only of their semantic representations, but also their syntactic representations. Traditional models of sentence processing hold that it is primarily the activation of syntactic representations that allows the meaning of words to be combine together to form a higher-order meaning (e.g. to determine 'who does what to whom': the assignment of thematic roles) [14]. However, over the past few years, a growing body of research suggests that comprehension is also driven by semantic combinatorial processes, including semantic memory-based processes, that, in some cases, can challenge even an unambiguous syntax [15]. We have suggested that the fine balance between semantic memory-based and syntactically-driven combinatorial processing is disrupted in schizophrenia [15], and that such a disruption can lead to errors in the build-up of higher-order meaning under some circumstances [16, 17]. There are several sources of evidence for this account $[1,2]$. Here I describe a couple of example ERP experiments.

The first experiment, carried out in collaboration with Dean Salisbury's lab, pitted an implausible overall sentence meaning against coherent semantic relationships between individual words. Sitnikova, Salisbury et al. [18] presented patient and controls with incongruous sentences like "The guests played bridge because the river had rocks in it" and congruous sentences like "Diving was forbidden from the bridge because the river had rocks in it." ERPs were measured to critical words such as "river" that, in all sentences, were semantically related to the dominant meaning of the preceding homograph ("bridge" meaning overpass). Whereas healthy controls showed an increase of the N400 to "river" when preceded by an incongruous relative to a congruous sentence context, patients failed to show this N400 modulation. Importantly, the very same patients did show a significant increase in N400 to incongruous words that were semantically unrelated to all preceding words in the sentence (e.g., when "cracks" were substituted for "rocks" in the above sentences). We interpreted these findings as suggesting that, in these patients, online neural processing was dominated by the semantic associative priming (between the homographs' dominant meaning and the critical word, "river"), at the expense of combining syntactic and semantic information to build up the sentence's overall context.

A second experiment directly pitted highly associative semantic relationships between a critical verb and its preceding content words ("breakfast", "eggs" and "eat") against the syntactically-dictated implausible interpretation ("Every morning at breakfast the eggs would eat..."). In healthy individuals, this conflict on "eat" leads to an attenuated N400 (a temporary 'semantic illusion'), followed by a later P600 effect that we have suggested reflects continued (mainly syntactically-driven) combinatorial attempts to make sense of the sentence $[15,19-21]$. In schizophrenia, however, no increased P600 is produced on "eat" in these sentences [17]. In other words, no online neural processing cost is incurred at all, despite the incongruity of the sentence as a whole; in patients, processing (and, in some 
cases, even the final interpretation) of such sentences was effectively dominated purely by the semantic relationship between "breakfast", "eggs" and "eat" [17].

At a neuroanatomical level, normal syntactically-driven sentence comprehension is thought to be mediated through activity within a network distributed across superior and middle temporal and inferior frontal cortices [22], while semantic memory-based retrieval during sentence comprehension is thought to be mediated through activity within inferior temporal and inferior frontal cortices [23, 24]. When demands to make sense of a sentence as a whole are increased (such in anomalous sentences that require reanalysis, e.g. "...at breakfast the eggs would eat" or “...at breakfast the boys would eats..."), additional regions also seem to be recruited, including premotor, dorsolateral prefrontal and parietal cortices [24]. In healthy individuals, the precise neurocognitive operations reflected by this additional recruitment and how it interacts with activity within perisylvian and temporal-occipital networks during processing remains unclear. However, if, as we have suggested, the balance of activity between semantic memory-based and syntactically-driven routes to comprehension is disrupted in schizophrenia, this would predict a dissociation between patients and controls in the modulation of these networks, particularly when demands to make sense of a sentence as a whole are high (such as on sentence-final words). Recent findings in our lab support this hypothesis: when semantically anomalous sentence-final words are contrasted with nonviolated words, normal recruitment within temporal (and inferior frontal) cortices is observed, but activity within dorsolateral prefrontal and parietal cortices is abnormally modulated [25, 26]. In future studies, we plan to manipulate semantic associative strength between individual words within sentences to determine whether, when associative strength is high, patients, relative to controls, will show even greater recruitment of temporal cortices to such associates, just as for single word-pairs.

\section{Building Meaning Discourse}

Effective thought, comprehension and communication require us to go well beyond making sense of a single sentence [27]. To construct meaning across sentences, we must establish semantic links between the propositions conveyed in sentences. In healthy individuals, establishing such coherence is often reliant on being able to retrieve information stored within semantic memory that is not explicitly stated in the text, i.e. generating inferences. For example, to make sense of a simple three-sentence scenario such as "John and Jack had an argument. Jack got very angry. The next day John had bruises.”, we must retrieve, construct and integrate a causal inference - "Jack hit John." In ERP experiments, the cost of such processing is manifest immediately as an increased N400 on "bruises", relative to words in sentences that do not require such inferences [28]. Moreover, using fMRI, a large bilateral neural network, distributed across temporal, inferior and superior frontal cortices, is activated to inference-requiring words [29]. Schizophrenia patients fail to show the immediate neural cost of semantically integrating "bruises" into its preceding context (no modulation of the N400) [28,30]. However, they do judge the causal coherence of these scenarios similarly to controls, and fMRI findings demonstrated that they showed normal recruitment of temporal-frontal cortices [31], suggesting that they are able to generate inferences, perhaps at a later stage of processing.

Not all discourse, however, is coherent. Healthy individuals appear to have a threshold such that they generate inferences when appropriate, but, during normal comprehension, they do not necessarily attempt this when the input seems very incoherent. If, for example, the final sentence in the above scenario is completely unrelated to its context (e.g. "The next day John ate breakfast"), although healthy individuals show an immediate semantic integration cost (an increased N400) on the critical word ("breakfast") [28], they will generally fail to generate an inference and they do not lead to further recruitment of temporal-frontal cortices 
[29]. A very different pattern is observed in schizophrenia. Despite an immediate failure to incur a semantic cost on "breakfast" during online neural processing (no N400 effect) [28], patients, unlike controls, do appear to recruit several temporal and prefrontal regions to these unrelated scenarios [31]. We suggest that this that may reflect an inappropriate retrieval of information from semantic memory i.e. inappropriate inferencing, that might be linked to the illogical connections between ideas that characterizes psychosis.

Constructing meaning across sentences also requires us to establish referential cohesion, i.e. to connect multiple references to the same entity. First mentions of the entity are referred to as antecedents and subsequent mentions are termed anaphors. Encouragingly, in a recent study, we showed that schizophrenia patients are able to establish cohesive links between categorical anaphors and their antecedents, at least when the context is highly constrained and explicitly provided [32]. This, however, may not be the case when the anaphor is a pronoun or when it is referentially ambiguous and future studies will determine whether schizophrenia patients show neural abnormalities in establishing cohesion under such circumstances.

\section{Building meaning during real-world comprehension}

Above, we have illustrated, through studies of language, how interactions between semantic memory and the build-up of higher-order meaning may be imbalanced in schizophrenia. This model also has implications for real-world comprehension outside the language system. Using a novel paradigm [33], we have investigated how patients build meaning as they comprehend real-world events. In this paradigm, participants view short movie-clips with final scenes that depict violations of actions in everyday events, e.g. a man attempting to shave with a rolling pin, where the critical objects do not have the semantic properties to execute the target action (a rolling pin does not have the semantic features to carry out the action of shaving). In healthy individuals, such violated scenes evoke not only an (anteriorly distributed) N400 effect, relative to non-violated scenes, but also a Late Positivity effect [33, 34]. We have suggested that the Late Positivity evoked by such action-based violations reflects participants' continued attempts to make sense of such events by computing the relationship between objects, actors and action (determining 'who does what to whom') in an analogous manner to the analyses reflected by the P600 evoked by semantically violated verbs, that, as noted above, may reflect continued attempts to compute syntactic-thematic relationships around such verbs [15, 34, 35]. In schizophrenia, the N400 effect evoked by the violated, relative to the non-violated, visual scenes was modulated normally (indeed, in patients with behavioral and verbal disorganization symptoms, its modulation was increased). In contrast, patients showed a clear attenuation of the Late Positive/P600 effect $[36,37]$. Most interestingly, the reduction of this positivity correlated with patients' deficits in goal-directed behavior (and not with other symptoms), suggesting some specificity in its power to predict real-world impairments of goal-directed activity.

\section{Conclusions}

This overview of some of our own recent work illustrates how the study of language and semantics can give insights into how abnormalities of thought in schizophrenia might arises in the brain. I have focused on interactions between semantic memory and the build-up of sentence and discourse context in language and visual event comprehension. However, I believe that this general model of impaired interactions between memory-based and combinatorial processing may generalize to understanding impairments in other domains including emotional and social cognition (see [38-40] for examples of such applications). My hope is that the study of how meaningful individual stimuli are integrated into a meaningful whole will complement more reductionalist approaches to the cognitive 
neuroscience of schizophrenia, and will give additional insights into the mechanisms of thought and real-world function in this disorder.

\section{Acknowledgments}

The work conducted by Gina Kuperberg cited in this review was supported by NIMH (R01 MH071635), NARSAD (with the Sidney Baer Trust) and a Claflin Distinguished Scholars Award from Mass. General Hospital. The content is based on talks given at two Satellite Symposia at the 2006 ECNS (EEG and Clinical Neuroscience) Society Conference, Boston, MA: (1) "Beyond N400: Where recent functional and behavioral findings on language abnormalities in schizophrenia lead us"; (2) "Revealing the neural basis of cognitive dysfunction in schizophrenia using multiple neuroimaging techniques." I thank Tali Ditman, Tatiana Sitnikova and Daphne Holt for helpful comments on the manuscript.

\section{References}

1. Kuperberg, GR.; Ditman, T.; Kreher, DA.; Goldberg, T. Approaches to understanding language dysfunction in neuropsychiatric disorders: Insights from the study of schizophrenia. In: Wood, S.; Allen, N.; Pantelis, C., editors. Handbook of Neuropsychology of Mental Illness. Cambridge University Press; 2008.

2. Kuperberg GR, Kreher DA, Ditman T. What can event-related potentials tell us about language, and perhaps even thought, in schizophrenia? International Journal of Psychophysiology. Special Issue on Language and Psychophysiology. under review.

3. Bleuler, E. Dementia praecox, or the group of schizophrenias. New York: International Universities Press; 1911/1950.

4. Manschreck TC, Maher BA, Milavetz JJ, Ames D, Weisstein CC, Schneyer ML. Semantic priming in thought disordered schizophrenic patients. Schizophrenia Research. 1988; 1:61-66. [PubMed: 3154508]

5. Spitzer M, Weisker I, Winter M, Maier S, Hermle L, Maher BA. Semantic and phonological priming in schizophrenia. Journal of Abnormal Psychology. 1994; 103:485-494. [PubMed: 7930048]

6. Neely, JH. Semantic priming effects in visual word recognition: a selective review of current findings and theories. In: Besner, D.; Humphreys, GW., editors. Basic Processes in Reading and Visual Word Recognition. Erlbaum; Hillsdale, NJ: 1991. p. 264-333.

7. Spitzer M, Braun U, Hermle L, Maier S. Associative semantic network dysfunction in thoughtdisordered schizophrenic patients: direct evidence from indirect semantic priming. Biological Psychiatry. 1993; 34:864-877. [PubMed: 8110913]

8. Mathalon DH, Faustman WO, Ford JM. N400 and automatic semantic processing abnormalities in patients with schizophrenia. Archives of General Psychiatry. 2002; 59(7):641-648. [PubMed: 12090817]

9. Kreher DA, Holcomb PJ, Kuperberg GR. An electrophysiological investigation of indirect semantic priming. Psychophysiology. 2006; 43(6):550-63. [PubMed: 17076811]

10. Kreher, DA.; Holcomb, PJ.; Goff, D.; Kuperberg, GR. Schizophrenia Bulletin. Increased neural semantic priming in schizophrenic thought disorder: evidence from event-related potentials. Advance Access published September 28, 2007

11. Kutas M, Hillyard SA. Reading senseless sentences: Brain potential reflect semantic incongruity. Science. 1980; 207:203-205. [PubMed: 7350657]

12. Kuperberg GR, Lakshmanan BM, Greve DN, West WC. Task and semantic relationship influence both the polarity and localization of hemodynamic modulation during lexico-semantic processing. Human Brain Mapping. In press.

13. Kuperberg G, Deckersbach T, Holt D, Goff D, West WC. Increased temporal prefrontal activity to semantic associations in schizophrenia. Archives of General Psychiatry. 2007; 64:138-151.

[PubMed: 17283282]

14. Osterhout, L.; Kim, A.; Kuperberg, GR. The Neurobiology of sentence comprehension. In: Spivey, M.; Joannisse, M.; McRae, K., editors. The Cambridge Handbook of Psycholinguistics. Cambridge University Press; Cambridge: 2007. 
15. Kuperberg GR. Neural mechanisms of language comprehension: Challenges to syntax. Brain Res, Special Issue: Mysteries of Meaning. 2007; 1146:23-49.

16. Kuperberg GR, Kreher DA, Goff D, McGuire PK, David AS. Building up linguistic context in schizophrenia: evidence from self-paced reading. Neuropsychology. 2006; 20(4):442-52. [PubMed: 16846262]

17. Kuperberg GR, Sitnikova T, Goff D, Holcomb PJ. Making sense of sentences in schizophrenia: electrophysiological evidence for abnormal interactions between semantic syntactic processing. Journal of Abnorm Psychol. 2006; 115(2):243-256.

18. Sitnikova T, Salisbury DF, Kuperberg G, Holcomb PI. Electrophysiological insights into language processing in schizophrenia. Psychophysiology. 2002; 39(6):851-860. [PubMed: 12462512]

19. Kuperberg GR, Sitnikova T, Caplan D, Holcomb PJ. Electrophysiological distinctions in processing conceptual relationships within simple sentences. Cogn Brain Res. 2003; 17(1):117129.

20. Kuperberg G, Caplan D, Sitnikova T, Eddy M, Holcomb P. Neural correlates of processing syntactic semantic thematic relationships in sentences. Language and Cognitive Processes. 2006; 21(5):489-530.

21. Kuperberg G, Kreher DA, Sitnikova T, Caplan D, Holcomb PJ. The role of animacy thematic relationships in processing active English sentences: Evidence from event-related potentials. Brain and Language. 2007; 100(3):223-238. [PubMed: 16546247]

22. Caplan D, Chen E, Waters G. Task-dependent and task-independent neurovascular responses to syntactic processing. Cortex. In press.

23. Kuperberg GR, Holcomb PJ, Sitnikova T, Greve D, Dale AM, Caplan D. Distinct patterns of neural modulation during the processing of conceptual syntactic anomalies. Journal of Cognitive Neuroscience. 2003; 15(2):272-293. [PubMed: 12676064]

24. Kuperberg GR, Lakshmanan TSBM. Neuroanatomical distinctions within the semantic system during sentence comprehension: Evidence from functional magnetic resonance imaging. NeuroImage. In press.

25. Kuperberg GR, Lakshmanan BM, Goff D, West WC. Double dissociations in hemodynamic modulation during the build-up of concrete and abstract meaning in schizophrenia: Evidence from fMRI. Schizophrenia Research Supplement. 2007 Abstract.

26. Kuperberg GR, West WC, Goff D, Lakshmanan B. fMRI reveals neuroanatomical dissociations during semantic integration in schizophrenia. Under review.

27. Zwaan RA, Radvansky GA. Situation models in language comprehension memory. Psychol Bull. 1998; 123(2):162-85. [PubMed: 9522683]

28. Ditman T, Kuperberg G. The time course of building discourse coherence in schizophrenia: an ERP Investigation. Psychophysiology. 2007; 44:991-1001. [PubMed: 17666031]

29. Kuperberg GR, Lakshmanan BM, Caplan DN, Holcomb PJ. Making sense of discourse: An fMRI study of causal inferencing across sentences. NeuroImage. 2006; 33(1):343-361. [PubMed: 16876436]

30. Paczynski M, Ditman T, Okano K, Kuperberg GR. Drawing inferences during discourse comprehension: An ERP Study. Cogn Neurosci Suppl. 2007

31. Kuperberg GR, Gulabani D, Goff D, Blais K, Caplan D, Holcomb PJ. From the Knight to the Right: an event-related fMRI study of schizophrenic thinking. Human Brain Mapping. 2005; (Suppl) Abstract.

32. Ditman T, Kuperberg GR. An ERP examination of lexico-semantic and contextual influences across sentence boundaries in schizophrenia. Journal of Cognitive Neuroscience Suppl. 2008 Abstract.

33. Sitnikova T, Kuperberg GR, Holcomb P. Semantic integration in videos of real-world events: an electrophysiological investigation. Psychophysiology. 2003; 40:160-164. [PubMed: 12751813]

34. Sitnikova T, Holcomb PJ, Kuperberg GR. Two neurocognitive mechanisms of semantic integration during the comprehension of visual real-world events. J Cogn Neurosci. In press.

35. Sitnikova, T.; Holcomb, P.; Kuperberg, GR. Neurocognitive mechanisms of human comprehension. In: Shipley, TF.; Zacks, JM., editors. Understanding Events: How Humans See, Represent, and Act on Events. Oxford University Press; In press 
36. Sitnikova T, Cotton J, West C, Holcomb P, Kuperberg G. Comprehension of real-world events in schizophrenia: an electrophysiological perspective. J Cogn Neurosci Suppl. 2005

37. Sitnikova T, Goff D, Kuperberg GR. Comprehending the real world in schizophrenia: distinct neurocognitive abnormalities dissociate disorganization and negative symptoms. Under review.

38. Holt, DJ.; Lynn, S.; Kuperberg, GR. Comprehending the emotional meaning of sentences: an ERP Study. Under review

39. Holt DJ, Lakshmanan BM, Freudenreich O, Goff DC, Rauch SL, Kuperberg GR. An event-related functional MRI study of neural responses to social information in schizophrenia. The EEG and Clinical Neuroscience Society meeting. 2006 Abstract.

40. Holt DJ, Lakshmanan B, Freudenreich O, Goff DC, Rauch SL, Kuperberg GR. Abnormal activation of the medial prefrontal and posterior cingulate cortices during affective appraisal in schizophrenia: relationship to delusions. Under review. 